\title{
Analytical modelling of single-walled carbon nanotube energies: the impact of curvature, length and temperature
}

\author{
Daniel Hedman ${ }^{1}$ (D) . J. Andreas Larsson ${ }^{1}$ D
}

Received: 10 October 2019 / Accepted: 30 January 2020 / Published online: 8 February 2020

(c) The Author(s) 2020 OPEN

\begin{abstract}
Recent breakthroughs in the field of single-walled carbon nanotube (SWCNT) growth have been achieved by combining theoretical models with experiments. Theoretical models rely on accurate energies for SWCNTs, obtained via first principle calculations in the form of density functional theory (DFT). Such calculations are accurate, but time and resource intensive which limits the size and number of systems that can be studied. Here, we present a new analytical model consisting of three fundamental energy expressions, parametrized using DFT, for fast and accurate calculation of SWCNT energies at any temperature. Tests against previously published results show our model having excellent accuracy, with an root mean square error in total energies below $2 \mathrm{meV}$ per atom as compared to DFT. We apply the model to study SWCNT growth on Ni catalysts at elevated temperatures by investigating the SWCNT/catalyst interface energy. Results show that the most stable interface shifts towards chiral edges as the temperature increases. The model's ability to perform calculations at any temperature in combination with its speed and flexibility will allow researcher to study more and larger systems, aiding future research into SWCNT growth.
\end{abstract}

Keywords Single-walled carbon nanotubes - Density functional theory · Analytical modelling · Curvature - Length . Temperature $\cdot$ Chirality $\cdot$ Energy

\section{Introduction}

Single-walled carbon nanotubes (SWCNTs) are hollow cylindrical tubes with the tube wall made up of a single layer of carbon atoms. Nanotubes have remarkable electrical, optical, thermal and mechanical properties that are dependent on the structure of the tube wall, known as the chirality of the SWCNT [1]. The chirality, and thus the properties of a SWCNT, is set during the creation (growth) of the tube. Today's methods for producing SWCNTs, catalytic chemical vapour deposition (CCVD), give products with a mixture of chiralities and thus a mixture of properties $[2$, 3]. This is one of the major problems holding back the use of nanotubes in commercial products. But in recent years, a great deal of progress has been made towards explaining the chirality of SWCNTs observed in experimental growth products.

The group of Yakobson related the number of axial screw dislocations (kinks) at the SWCNT-edge to their growth rate [4] and later expanded on this to give a continuum model of SWCNT growth [5]. The works of Hedman et al. [6] link experimentally determined chiralities from CCVD products to the stability of short SWCNTs produced at the early stages of growth. Whereas the diameter range of the produced tubes is controlled by the size of the catalytic particles used during synthesis. This group's

Electronic supplementary material The online version of this article (https://doi.org/10.1007/s42452-020-2139-z) contains supplementary material, which is available to authorized users.

Daniel Hedman, daniel.hedman@ltu.se; J. Andreas Larsson, andreas.1.larsson@ltu.se $\mid{ }^{1}$ Applied Physics, Division of Materials Science, Department of Engineering Sciences and Mathematics, Luleå University of Technology, 97187 Luleå, Sweden. 
recent paper relates the stability of SWCNTs to their length and shows a switch in the most stable chirality occurring (from armchair to zigzag) depending on the length of the SWCNT [7]. Recently, Magnin et al. [8] developed a thermodynamic model based on the configurational entropy of SWCNT-edges (the difference within the same chirality) to predict chiral distributions from CCVD experiments.

What these models have in common is that they rely on having accurate energies for SWCNTs and their terminations (hydrogen, catalyst metal, SWCNT-cap, etc.) to explain the chiral distributions found in experiments. These energies are usually calculated using density functional theory (DFT), which gives very accurate energies. However, calculations using DFT require access to large amount of computer resources and time. If a simple analytical model for calculating total energies of terminated SWCNTs was available, that eliminates the use of explicit DFT calculations. It would allow expansion of these models to include larger diameter tubes, longer tubes and larger span of chiralities. The inclusion of an entropy term would also allow for studies at elevated temperatures. Here, we present such a model.

Our analytical model, parametrized against DFT data, shows excellent agreement with DFT computed energies with a root mean square error (RMSE) in total energies below $2 \mathrm{meV} /$ atom. Applying the model to study catalytic growth of SWCNTs, we find that the most stable interface shifts towards chiral tubes as the growth temperature increases, from armchair towards zigzag for $\mathrm{H}$-terminated tubes, and from zigzag towards armchair for SWCNTs grown on Ni catalysts.

\section{Methodology}

One can think of the total energy for a SWCNT as consisting of three fundamental energies $E_{w}, E_{i}$ and $E_{t} . E_{w}$ is the energy per carbon atom of the tube wall. $E_{i}$ is the interface energy per bond (between the tube edge and the termination). This energy can be thought of as the relative binding energy between the carbon-carbon bonds and the carbon-termination bonds. Final, $E_{t}$ is the energy per atom of the SWCNT termination. These energies are multiplied by the number of atoms $\left(N_{w}, N_{t}\right)$ or bonds $\left(N_{i}\right)$ of each type and added to give the total energy of a SWCNT as

$E=N_{w} \cdot E_{w}+N_{i} \cdot E_{i}+N_{t} \cdot E_{t}$.

For finite SWCNTs, it is convenient to describe their length in number of layers, $S$, as described previously [7]. Using this definition, each layer, $S$, will contain $2(n+m)$ carbon atoms, where $n$ and $m$ are the chiral indices of the SWCNT. One can calculate the real length, $L$, (in units of $\AA$ ) of any finite SWCNT with a length of $S$ number of layers. Using the following equation (for derivation see the supplementary material),

$L=\frac{I_{c c}(n(3 S+1)+m(3 S-1)-3 d)}{2 \sqrt{n^{2}+m^{2}+n m}}$.

Here, $I_{\mathrm{cc}}$ is the average carbon-carbon bond length of SWCNTs ( $1.44 \AA$ ) and $d$ is the greatest common divisor of the chiral indices $d=\operatorname{gcd}(n, m)$.

A finite SWCNT of chirality $(n, m)$ and length $S$ has $N_{w}=2(n+m) \cdot S$ number of wall atoms and $N_{i}=2(n+m)$ edge bonds. If all the layers are full and the number of atoms in the termination equals the number edge bonds, e.g. $N_{t}=N_{i}$, then Eq. (1) can be written as

$E=\left(E_{w} \cdot S+E_{i}+E_{t}\right) \cdot 2(n+m)$.

It has been shown [9] that the energy per carbon atom of the tube wall can be described by the energy per atom of a flat graphene sheet, modified by a curvature term, $\frac{1}{R^{\prime}}$ as shown below

$E_{w}=\gamma \cdot\left(\frac{1}{R}\right)^{2}+\epsilon_{w}$.

Where $\epsilon_{w}$ is the energy per atom of a graphene sheet and $\gamma$ is the energy penalty term due to the curvature of the tube.

The edge of a SWCNT with chirality $(n, m)$ consists of $2 m$ carbon atoms with armchair configuration and $n-m$ atoms with zigzag configuration. One can, by using an expression similar to the one used to describe edge energies of graphene [10], write an expression for the interface energy per bond, $E_{i}$, of a SWCNT with any chirality. As a combination of the armchair, $E_{i}^{a c}$, and zigzag, $E_{i}^{z z}$ interface energies. This expression includes an armchair/zigzag mixing term, $E_{\xi}$, similar to [10] that describes the junction between edge atoms of armchair and zigzag configuration. The group of Bichara introduced a term for the configurational entropy of the SWCNT-edge [8]. Adding this term, $E_{T S}$, allows for the inclusion of temperature effects on the interface energy. Of course for direct comparison with DFT energies, this term should be set to zero. Using these terms, we arrive at an expression for the interface energy of any SWCNT with chirality $(n, m)$ as

$E_{i}=\frac{2 m}{n+m} E_{i}^{a c}+\frac{n-m}{n+m} E_{i}^{z Z}+E_{\xi}-E_{T S}$.

Similarly to the energy per carbon atom in the tube wall, $E_{w}$, the interface energies ( $E_{i}^{a c}$ and $E_{i}^{z z}$ ) will depend on the curvature of the SWCNT. However, unlike $E_{w}$ they are also dependent on the length of the tube. Since the finite length of the SWCNT leads to quantum confinement 
effects like to those observed for graphene nanoribbons (GNRs) [11, 12]. Due to these finite size effects, we use an expression similar to Eq. (4) but with an added length dependency term $\frac{1}{s}$ in order to describe the interface energies

$E_{i}^{a c}=\alpha^{a c} e^{\frac{\beta^{a c}}{R^{a c}}} \cdot\left(\frac{1}{S}\right)^{2}+\gamma^{a c} \cdot\left(\frac{1}{R^{a c}}\right)^{2}+\epsilon_{i}^{a c}$,

$E_{i}^{z z}=\alpha^{z z} e^{\frac{\beta^{z z}}{R z}} \cdot\left(\frac{1}{S}\right)^{2}+\gamma^{z z} \cdot\left(\frac{1}{R^{z z}}\right)^{2}+\epsilon_{i}^{z z}$.

Here, $\alpha^{a c}, \alpha^{z z}$ are energy penalty terms due to confinement effects arising from the finite length of the SWCNT. These are found to vary slightly depending on the curvature of

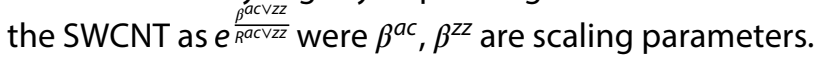

The curvature dependency for the interface energy is described by the parameters $\gamma^{a c}, \gamma^{z z}$. Finally, $\epsilon_{i}^{a c}$ and $\epsilon_{i}^{z z}$ are interface energies for graphene nanoribbons with armchair or zigzag edges. These energies can be calculated from DFT total energies using the following equation, derived from Eq. (1)

$\epsilon_{i}^{a c \mathrm{VZz}}=\frac{\epsilon_{\mathrm{DFT}}^{a \mathrm{cVZZ}}-N_{w} \cdot \epsilon_{w}-N_{t} \cdot E_{t}}{N_{i}}$.

Where $\epsilon_{\mathrm{DFT}}^{a c \mathrm{VZ} z}$ is the DFT calculated total energy of a GNR with armchair or zigzag edges. $\epsilon_{w}$ is the energy per atom of graphene [same as in Eq. (4)] and $E_{t}$ is the energy per atom for the termination of the GNR. It is obvious from Eq. (8) that the interface energy will depend on the termination of the GNR.

The well-known expression for the diameter of a SWCNT $[13,14]$ can be used in Eq. (4) to calculate the curvature

$\frac{1}{R}=\frac{2 \pi}{\sqrt{3} I_{\mathrm{cc}} \sqrt{n^{2}+m^{2}+n m}}$.

For Eqs. (6) and (7), this expression can be modified to give the radius of armchair or zigzag tubes for any given nanotube series $(n+m)$

$$
\begin{aligned}
& \frac{1}{R^{a c}}=\frac{1}{R}\left\{n=\frac{(n+m)}{2}, m=n\right\}=\frac{4 \pi}{3 I_{\mathrm{cc}}(n+m)}, \\
& \frac{1}{R^{z z}}=\frac{1}{R}\{n=(n+m), m=0\}=\frac{2 \pi}{\sqrt{3} I_{\mathrm{cc}}(n+m)} .
\end{aligned}
$$

The armchair/zigzag mixing term, $E_{\xi}$, in Eq. (5) consists of a combination of the number of armchair and zigzag atoms, $2 m$ and $n-m$, respectively. Multiplied by the ratio of armchair pairs $m / n$ and scaled with the total number of edge atoms $(n+m)$ to give
$E_{\xi}=\xi \cdot \frac{2 m \cdot(n-m)}{(n+m)^{2}} \cdot \frac{m}{n}$.

Where $\xi$ is the mixing energy parameter derived from DFT calculation on GNRs with different chiralities, see supplementary material for details (Fig. S3).

Finally, the energy corresponding to the configurational entropy of the SWCNT-edge at temperature $T$ is given by [8]

$E_{T S}=\frac{k_{B} T}{n+m} \ln \left(\frac{n !}{m !(n-m) !}\right)$.

With a slight modification since the original expression is given for the whole edge rather then per edge atom. Here, $k_{B}$ is the Boltzmann constant and $T$ the temperature in Kelvin.

\subsection{Model parameters}

The analytical model, as described above, contains 12 parameters. Out of these twelve, eleven are obtained from fitting against DFT data and one, $E_{t}=-3.386 \mathrm{eV}$ is derived directly from DFT calculations on a single $\mathrm{H}_{2}$ molecule. Note that this value of $E_{t}$ is only relevant for hydrogen terminated nanotubes. Parameter fitting was done using the MATLAB R2017a curve fitting toolbox with the Levenberg-Marquardt algorithm $[15,16]$. The DFT datasets used for fitting consist of previously published calculations [7] together with a few additional calculations. These calculations were performed using VASP [17-20] version 5.3.5 with the Perdew-Burke-Ernzerhof (PBE) exchangecorrelation functional. The VASP settings were the same as for the rest of the DFT datasets, for details see the supplementary material. All DFT datasets used for fitting are available through the NOMAD repository [21-24]. The resulting value for each parameter, obtained via fitting, is listed in Table 1. For details on the fitting process, see supplementary material Figs. S1, S2 and S3.

\section{Results and discussion}

The final expression for the total energy of $\mathrm{H}$-terminated SWCNTs Eqs. (3)-(7), (9)-(12) with $T=0 \mathrm{~K}$ and the parameter values from Table 1 is tested against previously published DFT calculations [6]. As seen in Fig. 1, the model agrees well with DFT calculated energies. The RMSE in the interface energy is $\sim 11 \mathrm{meV}$ per bond, the RMSE for the energy of the atoms in the tube wall is $\sim 1.9 \mathrm{meV}$ per atom, and for the total energy the RMSE is $\sim 1.6 \mathrm{meV}$ per atom. The error in the interface energy, $\delta_{i}$, is expected to be large since it accumulates the errors $\left(\delta_{w}, \delta_{t}\right)$ of $E_{w}$ and 
Table 1 Model parameters obtained from fitting Eqs. (4), (6) and (7) to the DFT dataset, see supplementary material Figs. S1, S2 and S3 for details

\begin{tabular}{cccl}
\hline Eq. & Param. & Value & Unit \\
\hline (4) & $\gamma$ & $2.038 \mathrm{eV} \AA^{2}$ \\
& $\epsilon_{w}$ & $-9.230 \mathrm{eV}$ \\
(6) & $\alpha^{a c}$ & $0.043 \mathrm{eV}$ \\
& $\beta^{a c}$ & 4.4 & $\AA$ \\
& $\gamma^{a c}$ & -1.2 & $\mathrm{eV} \AA^{2}$ \\
& $\epsilon_{i}^{a c}$ & $0.074 \mathrm{eV}$ \\
(7) & $\alpha^{z z}$ & -0.42 & $\mathrm{eV}$ \\
& $\beta^{z z}$ & -0.21 & $\AA$ \\
& $\gamma^{z z}$ & $0.023 \mathrm{eV} \AA^{2}$ \\
& $\epsilon_{i}^{z z}$ & $0.22 \mathrm{eV}$ \\
(12) & $\xi$ & $-0.20 \mathrm{eV}$ \\
\hline
\end{tabular}

$E_{t}$ as follows, $\delta_{i} \propto \delta_{w} \cdot S+\delta_{t}$ (see supplementary material). Assuming that $\delta_{w} \cdot S \gg \delta_{t}$, then a calculation using the RMSE for the tube wall energy gives $\delta_{i} \approx 1.9 \cdot 6=11.4$ meV per bond which is in good agreement with Fig. 1 a. The data for which the model was verified against [6] were not used for fitting of any parameters $\left(\alpha^{a c}, \alpha^{z z}, \beta^{a c}, \beta^{z z}, \gamma\right.$, $\gamma^{a c}, \gamma^{z z}, \epsilon_{w}, \epsilon_{i}^{a c}, \epsilon_{i}^{z z}, \xi$ or $\left.E_{t}\right)$. Hence, the agreement of the model with DFT calculations is impressive.
One can use the total energy model presented here to calculate energies for any $\mathrm{H}$-terminated SWCNTs using four variables the chiral indices $n, m$, the length $S$ and the temperature $T$. To further verify the model, we first calculate total energies for all tube chiralities in the 10-series around the switching length ( $S=3-13$ layers) with DFT and our analytical model (at $T=0 \mathrm{~K}$ ), to create $2 \mathrm{D}$ heat map of SWCNT stabilities, see Fig. 2. Here, we use the chiral angle, $\theta=\arctan \left(\frac{\sqrt{3} m}{m+2 n}\right)$, defined by a given $n$ and $m$. From this figure, it is clear that the analytical model agrees well with the results obtained from DFT. From the stability heat map in Fig. 2b, one can see that for short tube lengths the armchair chirality $\left(\theta=30^{\circ}\right)$ is the most stable. As the length of the SWCNT increases, the most stable chirality switches from armchair to zigzag $\left(\theta=0^{\circ}\right)$.

The switching length $\left(S^{*}\right)$ can be determined from Fig. $2 \mathrm{~b}$ to be 5.5 layers in accordance with previously published results [7]. This switch in stability can be explained by competing interface and tube wall energies. Equation 3 shows that the total energy of a SWCNT at short lengths is dominated by the interface and termination energies. As seen in Fig. S2, the armchair interface energy is lower than the zigzag interface energy, in agreement with previous

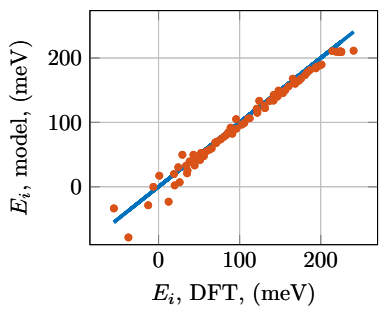

(a)

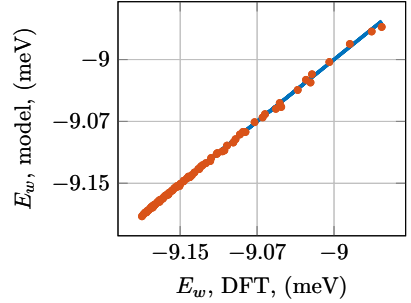

(b)

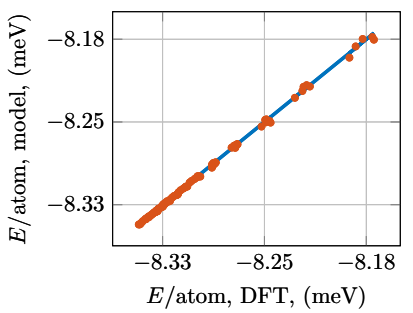

(c)
Fig. 1 The model at $T=0 \mathrm{~K}$ compared with DFT calculated energies of $\mathrm{H}$-terminated six-layer SWCNTs with all chiralities from the 8 to 20-series [6]. The red dots are the energies given by the model, and the blue line corresponds to DFT energies. $\mathbf{a}$ is the interface energy Eq. (5); here, the RMSE is $11.29 \mathrm{meV}$ per bond. b The energy per carbon atom of the tube wall Eq. (4), with an RMSE of $1.88 \mathrm{meV}$ per atom. c The total energy Eq. (3), here the RMSE is $1.61 \mathrm{meV}$ per atom
Fig. 2 Stability of H-terminated SWCNTs as a function of length $S$ (number of layers), for all chiralities from the 10-series. a SWCNT stabilities calculated using DFT energies, b stabilities calculated with the model at $T=0 \mathrm{~K}$. Here, $\theta=30^{\circ}$ are armchair and $\theta=0^{\circ}$ zigzag tubes. Both $\mathbf{a}$ and $\mathbf{b}$ have the same colorbar

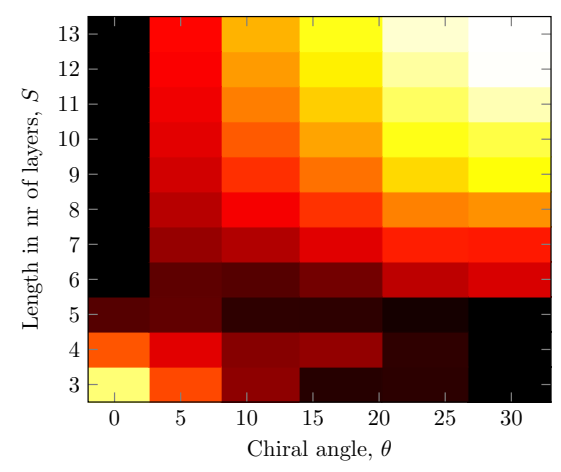

(a)

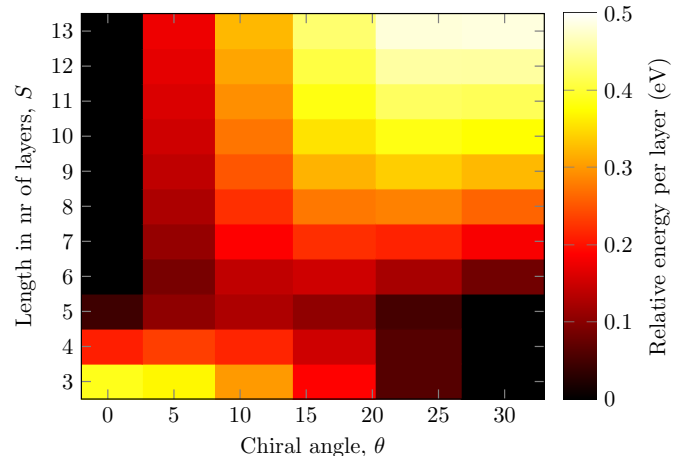

(b) 
studies [25-27]. Thus, for short armchair and zigzag tubes with the same termination the armchair tube has a lower energy. As the length of the tube increases, the energy of the atoms in the tube wall becomes dominant and since the zigzag tube has a lower curvature (lower tube wall energy), there will be a switch in the most stable chirality as the length of the SWCNT increases. To calculate all the tubes in the 10-series ( 6 unique chiralities) for 11 different lengths containing between 80 and 280 atoms with DFT can be done if needed. But we show that our analytical model can be used to calculate similar issues for any, or all SWCNT series.

Since the model is analytical, it does not limit the size of SWCNTs that can be calculated. Thus, as an example we calculate the switching length of very large tubes containing up to 9000 atoms. As seen in Table 2, the switching length increases rapidly as the SWCNT series increases (the diameter of the tubes increases). The long switching lengths $(>4 \mathrm{~nm}$ ) for large SWCNT series make reconfiguration from armchair to zigzag during growth unlikely for large diameter SWCNTs. However, due to the short switching length of small SWCNT series it might be possible to stimulate a transition from armchair to zigzag during the early stages of growth [28].

\subsection{Temperature effects on catalytic growth of SWCNTs}

The configurational entropy term, $E_{T S}$, allows us to study temperature effects on the interface energy by using
Eq. (1). Setting $N_{w}=N_{t}=0, N_{i}=(n+m)$ together with Eqs. (5), (12), (13), we obtain

$$
\begin{aligned}
F= & 2 m E_{i}^{a c}+(n-m) E_{i}^{z z}+\xi \cdot \frac{2 m^{2} \cdot(n-m)}{n(n+m)} \\
& +k_{B} T \cdot \ln \left(\frac{n !}{m !(n-m) !}\right) .
\end{aligned}
$$

where $E_{i}^{a c}$ and $E_{i}^{z z}$ are given by Eqs. (6), (7), respectively. This expression will give the interface energy for a SWCNT-edge as a function of $n, m, S$ and $T$.

Figure 3a shows the chiral angle for the lowest interface energy at temperature $T$ and SWCNT series $(n+m)$ of a H-terminated edge obtained by minimizing Eq. (14) with respect to $n$ and $m$ using $S=4, \epsilon_{i}^{a c}=0.074 \mathrm{eV}$ and $\epsilon_{i}^{z z}=0.22 \mathrm{eV}$. The figure shows that at low temperatures the interface energy for a $\mathrm{H}$-terminated edge is the lowest for armchair and near armchair chiralities as expected from DFT calculations. When the temperature increases, the configurational entropy has a larger and larger effect on the interface energy causing it to decrease for chiral tubes. As seen in Fig. 3a, this causes the chirality of the most stable interface energy to shift towards lower chiral angles. Using the 16-series as an example, we see that the most stable chirality, $(8,8)$ at $0 \mathrm{~K}$, changes to $(9,7)$ above $161 \mathrm{~K}$ and to $(10,6)$ above $1515 \mathrm{~K}$. The $\mathrm{H}$-terminated SWCNT-edge strongly favourers armchair chirality over zigzag due to the large difference in the two interface energies $\epsilon_{i}^{a c}=0.074 \mathrm{eV}$ and $\epsilon_{i}^{z z}=0.22 \mathrm{eV}$. This is an interesting result on its own; however, it is hard to compare

Table 2 Calculated switching lengths, $S^{*}$, using the total energy model at $T=0 \mathrm{~K}$ for SWCNT series denoted by $(n+m)$

\begin{tabular}{lllllllll}
\hline$(n+m)$ & 10 & 13 & 16 & 20 & 25 & 32 & 40 & 50 \\
\hline$S^{*}$ & 5.5 & 7.5 & 11 & 16 & 23.5 & 37 & 56.5 \\
$L^{a c}(\mathrm{~nm})$ & 1.3 & 1.9 & 2.6 & 3.9 & 5.9 & 9.1 & 14.0 & 21.6 \\
\hline
\end{tabular}

Here, $L^{a c}$ is the real length calculated by Eq. (2) for armchair tubes with $S^{*}$ number of layers

Fig. 3 The chiral angle of the most stable interface (lowest interface energy) for $S=4$ (short tubes) as a function of temperature and SWCNT series. For a $\mathrm{H}$-terminated edge and $\mathbf{b}$ edge terminated with a Ni nanoparticle. The colorbar indicates the chiral angle with $\theta=30^{\circ}$ corresponding to armchair and $\theta=0^{\circ}$ zigzag. Both a and $\mathbf{b}$ have the same colorbar

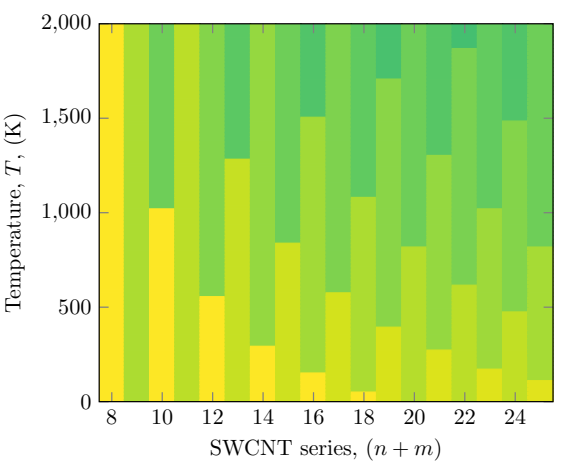

(a)

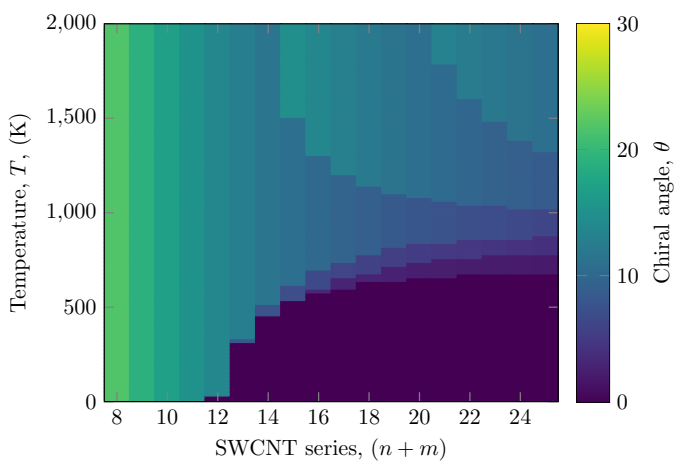

(b) 
$\mathrm{H}$-termination to actual metal nanoparticles used as catalysts for SWCNT growth.

To study the temperature effects on catalytic growth of SWCNTs, we use Eq. (14) together with armchair and zigzag interface energies for a $\mathrm{Ni}$ catalyst. These have been calculated to be $\epsilon_{i}^{a c}=0.321 \mathrm{eV}$ and $\epsilon_{i}^{z z}=0.234 \mathrm{eV}$ for a 55 metal Ni catalyst [29]. Minimizing Eq. (14) with respect to $n$ and $m$ using these interface energies and $S=4$ gives the chiral angle for the lowest interface energy at temperature $T$ and series $(n+m)$. As seen in Fig. 3b, the chirality for the most stable interface energy is largely unaffected by temperature for small SWCNT series $8 \leq n+m \leq 12$. But for larger series there is a shift in the most stable chirality from zigzag towards armchair (chiral tubes) as the temperatures increases. An example would be for the 16-series where the most stable chirality, $(16,0)$ at $0 \mathrm{~K}$, changes to $(15,1)$ above $576 \mathrm{~K}$, to $(14,2)$ above $604 \mathrm{~K},(13,3)$ above $692 \mathrm{~K}$ and to $(12,4)$ above $1303 \mathrm{~K}$. Also interesting is to look at the 13-series for which the most stable chirality at $873.15 \mathrm{~K}\left(600^{\circ} \mathrm{C}\right)$ is $(10,3)$. This chirality is very close to the experimental results by Chiang et al. [30] who obtained mostly $(9,4)$ tubes from SWCNT growth on floating Ni catalysts using $\mathrm{C}_{2} \mathrm{H}_{2}$ at $600^{\circ} \mathrm{C}$. Interestingly enough, the configuration entropy term must be included to reproduce the experimental results. Because DFT calculations exclusively predict zigzag tubes to have the most stable interface with Ni catalysts $[2,29,31]$. Since DFT calculations are at zero Kelvin, this means that a possible reason why theory (usually favouring the zigzag interface) and experiments (usually resulting in mostly armchair and near armchair SWCNT [6]) give different pictures may be the effect of temperature. But SWCNT growth is such a complicated process that it is likely that several effects combine to explain this difference between theory and experiment.

\section{Conclusions}

We present an analytical model, parametrized using DFT data, for calculating total energies of SWCNTs with any chirality $(n, m)$, length $S$ and at any temperature $T$. The model shows excellent agreement at $T=0 \mathrm{~K}$ with DFT calculations (RMSE $<2 \mathrm{meV} / \mathrm{atom}$ ) and can be expanded on to include SWCNTs with other terminations such as catalytic metals, SWCNT-caps. This is possible by calculating $\epsilon_{e}^{a c}, \epsilon_{e}^{z Z}$, $E_{t}$ using DFT and if necessary fit $\gamma, \gamma^{a c}, \gamma^{z z}$ and $\xi$ to a few GNR and SWCNT structures, with the desired terminations. Since the model is analytical, it allows for nearly instantaneous calculations of SWCNT energies as compared to the self-consistent approach of DFT. Allowing for the study of large SWCNTs with a wide range of chiralities, lengths and terminations.

SN Applied Sciences
The inclusion of the configurational entropy term enables calculation of SWCNT energies at elevated temperatures. This can be used to study the effects of temperature on the interface energy, which allows for prediction of SWCNT chiralities at experimental growth conditions. Our results show that as the temperature increases the most stable interface energy shifts towards chiral tubes, from armchair towards zigzag for $\mathrm{H}$-terminated tubes and from zigzag towards armchair for SWCNTs grown on $\mathrm{Ni}$ catalysts. Interestingly, DFT calculations show a thermodynamic preference towards zigzag chirality for tubes grown on Ni catalysts. But the inclusion of the configuration entropy term in our model shifts the thermodynamic preference towards near zigzag tubes in agreement with experiments.

The fast calculation of total energies at elevated temperatures will allow for wider searches of possible terminations (catalytic metals) that might be used for chiralityspecific growth of SWCNTs in addition to the prediction of chiral distributions in SWCNT growth experiments. Thus, this new model is a useful tool for future research into SWCNT growth.

Acknowledgements Open access funding provided by Luleå University of Technology. This work was performed at Luleå University of Technology, and the authors would like to acknowledge the computational resources and knowhow provided by the Swedish National Infrastructure for Computing (SNIC) at HPC2N and NSC.

Funding This study was funded by the Swedish Research Council, Knut and Alice Wallenberg Foundation, Kempe Foundations, Länsstyrelsen Norrbotten and Interreg Nord.

\section{Compliance with ethical standards}

Conflict of interest The author declares that they have no conflict of interest.

Open Access This article is licensed under a Creative Commons Attribution 4.0 International License, which permits use, sharing, adaptation, distribution and reproduction in any medium or format, as long as you give appropriate credit to the original author(s) and the source, provide a link to the Creative Commons licence, and indicate if changes were made. The images or other third party material in this article are included in the article's Creative Commons licence, unless indicated otherwise in a credit line to the material. If material is not included in the article's Creative Commons licence and your intended use is not permitted by statutory regulation or exceeds the permitted use, you will need to obtain permission directly from the copyright holder. To view a copy of this licence, visit http://creativecommons .org/licenses/by/4.0/.

\section{References}

1. Bati ASR, Yu L, Batmunkh M, Shapter JG (2018) Synthesis, purification, properties and characterization of sorted singlewalled carbon nanotubes. Nanoscale 10(47):22087. https://doi. org/10.1039/C8NR07379A 
2. Wang $X$, He M, Ding F (2018) Chirality-controlled synthesis of single-walled carbon nanotubes-From mechanistic studies toward experimental realization. Mater Today 21(8):845. https ://doi.org/10.1016/j.mattod.2018.06.001

3. Rao R, Pint $C L$, Islam $A E$, Weatherup RS, Hofmann $S$, Meshot ER, Wu F, Zhou C, Dee N, Amama PB, Carpena-Nuñez J, Shi W, Plata DL, Penev ES, Yakobson BI, Balbuena PB, Bichara C, Futaba DN, Noda S, Shin H, Kim KS, Simard B, Mirri F, Pasquali M, Fornasiero F, Kauppinen El, Arnold M, Cola BA, Nikolaev P, Arepalli $S$, Cheng HM, Zakharov DN, Stach EA, Zhang J, Wei F, Terrones M, Geohegan DB, Maruyama B, Maruyama S, Li Y, Adams WW, Hart AJ (2018) Carbon nanotubes and related nanomaterials: critical advances and challenges for synthesis toward mainstream commercial applications. ACS Nano 12(12):11756. https://doi. org/10.1021/acsnano.8b06511

4. Ding F, Harutyunyan AR, Yakobson BI (2009) Dislocation theory of chirality-controlled nanotube growth. Proc Natl Acad Sci 106(8):2506. https://doi.org/10.1073/pnas.0811946106

5. Artyukhov VI, Penev ES, Yakobson BI (2014) Why nanotubes grow chiral. Nat Commun 5:4892. https://doi.org/10.1038/ ncomms5892

6. Hedman D, Barzegar HR, Rosén A, Wågberg T, Larsson JA (2015) On the stability and abundance of single walled carbon nanotubes. Sci Rep 5:16850. https://doi.org/10.1038/srep16850

7. Hedman D, Larsson JA (2017) Length dependent stability of single-walled carbon nanotubes and how it affects their growth. Carbon 116:443. https://doi.org/10.1016/j.carbon.2017.02.007

8. Yann M, Hakim A, François D, Annick L, Christophe B (2018) Entropy-driven stability of chiral single-walled carbon nanotubes. Science 362(6411):212. https://doi.org/10.1126/scien ce.aat6228

9. Kudin KN, Scuseria GE, Yakobson BI (2001) C 2 F, BN, and C nanoshell elasticity from ab initio computations. Phys Rev $B$ 64(23):235406. https://doi.org/10.1103/PhysRevB.64.235406

10. Liu Y, Dobrinsky A, Yakobson BI (2010) Graphene edge from armchair to zigzag: the origins of nanotube chirality? Phys Rev Lett 105(23):235502. https://doi.org/10.1103/PhysRevLett.105.23550 2

11. Sako R, Hosokawa $H$, Tsuchiya $H$ (2011) Computational study of edge configuration and quantum confinement effects on graphene nanoribbon transport. IEEE Electron Device Lett 32(1):6. https://doi.org/10.1109/LED.2010.2086426

12. Kan E, Li Z, Yang J (2011) Graphene nanoribbons: geometric, electronic, and magnetic properties (IntechOpen, 2011). Phys Appl Graphene. https://doi.org/10.5772/14112

13. Saito R, Fujita M, Dresselhaus G, Dresselhaus MS (1992) Electronic structure of chiral graphene tubules. Appl Phys Lett 60(18):2204. https://doi.org/10.1063/1.107080

14. Dresselhaus MS, Dresselhaus G, Saito R (1995) Physics of carbon nanotubes. Carbon 33(7):883. https://doi.org/10.1016/00086223(95)00017-8

15. Levenberg K (1944) A method for the solution of certain nonlinear problems in least squares. Q Appl Math 2(2):164. https:// doi.org/10.1090/qam/10666

16. Marquardt $D$ (1963) An algorithm for least-squares estimation of nonlinear parameters. J Soc Ind Appl Math 11(2):431. https ://doi.org/10.1137/0111030
17. Kresse G, Hafner J (1993) Phys Rev B 47(1):558. https://doi. org/10.1103/PhysRevB.47.558

18. Kresse G, Furthmüller J (1996) Ab initio molecular dynamics for liquid metals. Comput Mater Sci 6(1):15. https://doi. org/10.1016/0927-0256(96)00008-0

19. Kresse G, Furthmüller J (1996) Efficient iterative schemes for ab initio total-energy calculations using a plane-wave basis set. Phys Rev B 54(16):11169. https://doi.org/10.1103/PhysR evB.54.11169

20. Kresse G, Joubert D (1999) From ultrasoft pseudopotentials to the projector augmented-wave method. Phys Rev B 59(3):1758. https://doi.org/10.1103/PhysRevB.59.1758

21. Hedman D, Larsson JA (2017) NOMAD repository: Graphene. https://doi.org/10.17172/NOMAD/2017.10.17-1

22. Hedman D, Larsson JA (2017) NOMAD repository: periodic armchair and zigzag SWCNTs, 8 to 20-series. https://doi. org/10.17172/NOMAD/2017.10.16-1

23. Hedman D, Larsson JA (2017) NOMAD repository: periodic armchair and zigzag GNRs. https://doi.org/10.17172/NOMAD /2017.10.17-2

24. Hedman D, Larsson JA (2017) NOMAD repository: finite armchair and zigzag SWCNTs, 8 to 20-series, 4 to 22-layers. https://doi. org/10.17172/NOMAD/2017.10.23-1

25. Koskinen P, Malola S, Hakkinen H (2008) Self-passivating edge reconstructions of graphene. Phys Rev Lett 101(11):115502. https://doi.org/10.1103/PhysRevLett.101.115502

26. Baran J, Kołodziejczyk W, Larsson P, Ahuja R, Larsson J (2012) On the stability of single-walled carbon nanotubes and their binding strengths. Theor Chem Acc 131(9):1. https://doi.org/10.1007/ s00214-012-1270-3

27. Li Y, Ahuja R, Larsson JA (2014) Communication: origin of the difference between carbon nanotube armchair and zigzag ends. J Chem Phys 140(9):091102. https://doi.org/10.1063/1.4867744

28. Zhao Q, Xu Z, Hu Y, Ding F, Zhang J (2016) Chemical vapor deposition synthesis of near-zigzag single-walled carbon nanotubes with stable tube-catalyst interface. Sci Adv 2(5):1. https://doi. org/10.1126/sciadv.1501729

29. Hedman D (2019) Single-walled carbon nanotubes: a theoretical study of stability, growth and properties. Ph.D. Thesis, Luleå University of Technology. https://doi.org/10.13140/RG.2.2.14638 .97602

30. Chiang WH, Sankaran RM (2009) Linking catalyst composition to chirality distributions of as-grown single-walled carbon nanotubes by tuning $\mathrm{Ni}(\mathrm{x}) \mathrm{Fe}(1-\mathrm{x})$ nanoparticles. Nat Mater 8(11):882. https://doi.org/10.1038/nmat2531

31. Ding $F$, Larsson $P$, Larsson JA, Ahuja R, Duan $H$, Rosén $A$, Bolton $\mathrm{K}$ (2008) The importance of strong carbon-metal adhesion for catalytic nucleation of single-walled carbon nanotubes. Nano Lett 8(2):463. https://doi.org/10.1021/nl072431m

Publisher's Note Springer Nature remains neutral with regard to jurisdictional claims in published maps and institutional affiliations. 